Check for updates

Cite this: RSC Adv., 2018, 8, 37729

Received 22nd August 2018

Accepted 9th October 2018

DOI: $10.1039 / c 8 r a 07043 a$

rsc.li/rsc-advances

\section{Rapid colorimetric glucose detection via chain reaction amplification of acrylic functionalized $\mathrm{AgCSiO}_{2}$ nanoparticles $\uparrow$}

\author{
Xiaofeng Lin, \$Wensheng Lin, \$Meijia Yang, Jiayao Chen, Dingshan Yu, (D) Wei Hong \\ and Xudong Chen 1 *
}

\begin{abstract}
The chain reaction amplification mechanism (CRAM) has been extensively studied, but it has not been effectively developed at the molecular scale and still needs to consume amounts of monomers to show the macroscopic phenomenon needed for detection. Herein, rationally-designed silica-coated silver nanoparticles with acrylic acid-functionalization were used as a plasmonic nanosensor to realize highly sensitive and fast colorimetric glucose detection with less monomer consumption, which effectively integrated CRAM with the localized surface plasmon resonance effect, developing CRAM at the molecular scale. The glucose detection mechanism of the proposed sensor was based on free-radical polymerization by biocatalytic initiation, which would induce the aggregation of Ag NPs, leading to a decrease in the plasmon resonance intensity. As a result, the detection limit could reach $2.06 \times 10^{-5} \mathrm{M}, 10$ times lower than that of a commercial glucose assay kit with a limit of $1.1 \times 10^{-4} \mathrm{M}$. Moreover, FDTD simulation further confirmed that the intensity of the extinction gradually decreased with an increase in the degree of aggregation of Ag NPs. The approach could be used for high selectivity toward glucose detection and would be suitable for other practical applications of the detection of low concentrations of glucose.
\end{abstract}

\section{Introduction}

Glucose, one of the most important sources of physiological energy, can be conducive to enhancing memory, stimulating calcium absorption and increasing cell-to-cell communication. ${ }^{1,2}$ However, an excessive concentration of blood glucose will lead to obesity and diabetes, while a low concentration of blood glucose will result in hypoglycemia or even worse. Meanwhile, glucose is essential to the maintenance of good brain functions: for example, a lower concentration of glucose has been found in the brain blood of Alzheimer's patients compared with other brains. ${ }^{3}$ As a consequence, it is extremely important to enhance the accuracy of glucose detection in order to more precisely monitor the glucose concentration in human blood. In previous reports, significant effort on the determination of glucose concentration has been focused on various methods, such as electrochemical sensing, ${ }^{4}$ fluorescent sensing, ${ }^{5}$ peroxidase mimics, ${ }^{6-11}$ and surface enhanced Raman

Key Laboratory for Polymeric Composite and Functional Materials of Ministry of Education of China, Guangdong Engineering Technology Research Center for High-performance Organic and Polymer Photoelectric Functional Films, School of Chemistry, Sun Yat-sen University, Guangzhou 510275, P. R. China. E-mail: cescxd@mail.sysu.edu.cn

$\dagger$ Electronic supplementary information (ESI) available. See DOI: 10.1039/c8ra07043a

$\ddagger$ X. F. Lin and W. S. Lin contributed equally to this work. spectroscopy (SERS). ${ }^{12}$ Among the aforementioned methods, SERS as a method of highly sensitive detection has attracted a surge in research attention in recent years. Nevertheless, difficulties derived from the precise preparation and repeatability of the reinforced substrate in SERS have hindered its practical application. In addition, although sensors based on SERS have made considerable progress on the aspect of qualitative detection, they still need further exploration in the field of quantitative detection..$^{13,14}$

Compared with the method of SERS, localized surface plasmon resonance (LSPR) could focus the light on the vicinity of the nanoparticles, resulting in the formation of an enhanced electromagnetic field on the surface of the nanoparticles. ${ }^{12,13}$ Such unique optical properties could be applied in numerous significant applications in various research fields, such as plasmon-assisted catalysis, ${ }^{15}$ solar cells, ${ }^{16,17}$ plasmonic sensors, ${ }^{18-20}$ the detection of single molecules ${ }^{21,22}$ luminescence enhancement, ${ }^{23,24}$ etc. In particular, owing to the low consumption and high sensitivity of the SPR peaks depended on the refractive index of the surrounding medium, ${ }^{25}$ the plasmonic sensors based on the LSPR effect could improve the SERS's problems of the substrate preparation and the quantitative detection. However, the sensitivity of sensors based on the LSPR effect still need to be further enhanced for the development of glucose detection.

In order to further improve this situation, the chain reaction amplification mechanism (CRAM) is an efficient way of using 
chain reactions to dramatically amplify the sensitivity of the sensing, ${ }^{26-28}$ which could reach the sensitivity level of femtomoles, or even zeptomoles. Moreover, CRAM could even be combined with an enzyme-linked immuno sorbent assay to further decrease the detection limit of biomolecules and is attracting extensive attention. ${ }^{29}$ However, traditional sensing based on CRAM systems needs to consume a large number of reactive monomers in order to present a macroscopic phenomenon via numerous double bond polymerizations.

Consequently, if the LSPR effect could be combined with the more sensitive detection method of CRAM, efficient sensors with less monomer consumption, lower detection time and higher sensitivity could be obtained. To this end, we have demonstrated a new class of plasmonic nanosensor based on acrylic acid-functionalized silica-coated silver nanoparticles, which effectively integrate CRAM with the LSPR effect, to realize highly sensitive and fast colorimetric glucose detection with less monomer consumption. The newly-developed sensor showed a limit of detection (LOD) of $2.06 \times 10^{-5} \mathrm{M}, 10$ times lower than that of a commercial glucose assay kit with a limit of $1.1 \times 10^{-4} \mathrm{M}$. The glucose detection mechanism of the proposed sensor was based on free-radical polymerization by biocatalytic initiation, which would induce the aggregation of $\mathrm{Ag}$ NPs and thereby cause a decrease in plasmon resonance intensity. Simultaneously, the detection sensitivity was strongly dependent on the degree of aggregation of the Ag NPs, which was confirmed by FDTD simulation. The approach could be used for high selectivity toward glucose detection and would be suitable for other practical applications of the detection of low concentrations of glucose.

\section{Experimental}

\section{Materials}

Silver nitrate $\left(\mathrm{AgNO}_{3}\right.$, purity $\left.>99.999 \%\right)$, trisodium citrate dihydrate $\left(\mathrm{Na}_{3} \mathrm{C}_{6} \mathrm{H}_{5} \mathrm{O}_{7} \cdot 2 \mathrm{H}_{2} \mathrm{O}\right.$, purity $\left.>99 \%\right)$, tetraethyl orthosilicate (TEOS, $\mathrm{Si}\left(\mathrm{C}_{2} \mathrm{H}_{5} \mathrm{O}\right)_{4}$, purity $>99 \%$ ), (3-aminopropyl)trimethoxysilane (APTMS, $\mathrm{C}_{6} \mathrm{H}_{17} \mathrm{NO}_{3} \mathrm{Si}$, purity >97\%), 1-ethyl-3-(3(dimethylamino)propyl)carbodiimide (EDC, $\mathrm{C}_{8} \mathrm{H}_{17} \mathrm{~N}_{3} \cdot \mathrm{HCl}$, purity $>98.0 \%$ ), $N$-hydroxysuccinimide (NHS, $\mathrm{C}_{4} \mathrm{H}_{5} \mathrm{NO}_{3}$, purity $>98 \%)$, acrylic acid $\left(\mathrm{C}_{2} \mathrm{H}_{3} \mathrm{COOH}\right.$, purity $\left.>99.5 \%\right)$, ferrous chloride $\left(\mathrm{FeCl}_{2}\right.$, purity $\left.>98 \%\right)$, ammonia water $\left(\mathrm{NH}_{4} \mathrm{OH}, 30 \mathrm{wt} \%\right)$, hydrogen peroxide $\left(\mathrm{H}_{2} \mathrm{O}_{2}\right)$, 5,5-dimethyl-1-pyrroline $\mathrm{N}$-oxide (DMPO, 97\%), glucose, ascorbic acid, D-fructose, $\beta$-cyclodextrin, lactose and maltose were purchased from Aladdin (China). The glucose assay kit, phosphate-buffered saline (PBS), fetal bovine serum and glucose oxidase (GOx) from Aspergillus niger were purchased from Sigma-Aldrich (United States). Ultrapure water was used in all experiments.

\section{Synthesis of silica-coated silver nanoparticles (Ag@SiO $\left.{ }_{2} \mathrm{NPs}\right)$}

The Ag@SiO ${ }_{2}$ NPs solution was synthesized according to previously published methods with slight modifications. ${ }^{30}$ Firstly, Ag NPs were prepared by dropwise addition of $10 \mathrm{~mL}$ of $38.8 \mathrm{mM}$ sodium citrate aqueous solution over $2 \mathrm{~min}$ into $490 \mathrm{~mL}$ of boiling aqueous solution containing $90 \mathrm{mg}$ of $\mathrm{AgNO}_{3}$ under vigorous stirring. After boiling for $1 \mathrm{~h}$, the heat was turned off and the reaction solution cooled to room temperature. ${ }^{31}$ The as-prepared silver colloid was centrifuged at $500 \mathrm{rpm}$ for $1 \mathrm{~h}$ to remove larger Ag NPs, and the remaining silver NPs in solution had an average size of $c a .50 \mathrm{~nm}$ by TEM. Then, $50 \mathrm{~mL}$ of as-obtained silver colloid were transferred into a roundbottom flask of $500 \mathrm{~mL}$ in volume containing ethanol (200 $\mathrm{mL}$ ), followed by adjustment of the $\mathrm{pH}$ value to $c a .10$ via the addition of $c a .30 \mathrm{wt} \%$ ammonia $(6.25 \mathrm{~mL})$. Subsequently, $4 \mathrm{~mL}$ of $10 \mathrm{mM}$ TEOS ethanol solution was added into the roundbottom flask over $2 \mathrm{~h}$ at time intervals of $30 \mathrm{~min}$ under vigorous shaking, and the resulting solution was further reacted for $24 \mathrm{~h}$ at a constant temperature of $30{ }^{\circ} \mathrm{C}$. $\mathrm{Ag} @ \mathrm{SiO}_{2} \mathrm{NPs}$ were collected by centrifugation at $10000 \mathrm{rpm}$ for $15 \mathrm{~min}$ and further washed with ethanol three times. Purified $\mathrm{Ag} @ \mathrm{SiO}_{2} \mathrm{NPs}$ were stirred at $60{ }^{\circ} \mathrm{C}$ for $2 \mathrm{~h}$ to improve their stability. The samples of purified $\mathrm{Ag} @ \mathrm{SiO}_{2}$ NPs with a certain thickness of silica ( $c a .10$ $\mathrm{nm})$ were redispersed into ethanol $(50 \mathrm{~mL})$ for further characterization and functionalization.

\section{Activation of the acrylic acid}

Acrylic acid $(100 \mu \mathrm{L})$ was added to deionized water $(10 \mathrm{~mL})$ containing EDC/NHS (5 mM), and incubated for at least for $2 \mathrm{~h}$ to ensure the acrylic acid was efficiently activated.

\section{Synthesis of acrylic acid-functionalized $\mathrm{Ag} @ \mathrm{SiO}_{2}$ NPs}

To obtain the amine-functionalized Ag@SiO ${ }_{2}$ NPs, firstly, the $\mathrm{Ag} @ \mathrm{SiO}_{2}$ solution $(50 \mathrm{~mL})$ was added into $1 \mathrm{~mL}$ of ethanol solution containing APTMS (10 $\mathrm{mM}$ in ethanol). Subsequently, the mixture was stirred for $16 \mathrm{~h}$ at a temperature of $50{ }^{\circ} \mathrm{C}$. The resulting nanoparticles were washed by centrifugation with ethanol and deionized water three times each in order to remove the unbound molecules of APTMS. Then, the aminefunctionalized $\mathrm{Ag} @ \mathrm{SiO}_{2}$ NPs were redispersed into activated acrylic acid solution $(10 \mathrm{~mL})$, and incubated for $2 \mathrm{~h}$ in order to obtain the acrylic acid-functionalized $\mathrm{Ag} @ \mathrm{SiO}_{2} \mathrm{NPs}$. After that, the acrylic acid-functionalized $\mathrm{Ag} @ \mathrm{SiO}_{2} \mathrm{NPs}$ were collected by centrifugation at $10000 \mathrm{rpm}$ for $15 \mathrm{~min}$ and further washed with deionized water three times, and finally redispersed into deionized water $(50 \mathrm{~mL})$ for further characterization.

\section{Characterization}

The absorption and extinction spectra in the range of 300$800 \mathrm{~nm}$ were measured using a UV-VIS-NIR spectrophotometer (UV3600, Shimadzu) with a $1 \mathrm{~cm}$ quartz cuvette. Transmission electron micrographs were measured by transmission electron microscopy (TEM, FEI Tecnai G2 Spirit) operating at an accelerating voltage of $120 \mathrm{kV}$. Electron spin resonance (ESR, Bruker A300) was used to test the existence of $\cdot \mathrm{OH}$ radicals.

\section{Sensor testing}

A series of glucose solutions of different concentrations were prepared to test the availability of the plasmonic sensors. In a typical test, $4 \mathrm{~mL}$ of acrylic acid-functionalized $\mathrm{Ag@SiO} \mathrm{NPs}_{2}$ solution was added to a $5 \mathrm{~mL}$ test tube, followed by the addition 
of $10 \mu \mathrm{L}$ of $\mathrm{Fe}^{\mathrm{II}}$ ions $(0.28 \mathrm{M}$ in deionized water at $\mathrm{pH} 4), 100 \mu \mathrm{L}$ of GOx $\left(2 \times 10^{-5} \mathrm{M}\right.$ in PBS) and $100 \mu \mathrm{L}$ of glucose solution (different concentrations in PBS). The mixture was vigorously shaken by mini-shakers at $800 \mathrm{rpm}$ for $3 \mathrm{~min}$. And the extinction spectra in the range of 300-800 nm were collected by a UV-VIS-NIR spectrophotometer using a quartz cuvette of $1 \mathrm{~cm}$. Additionally, in control experiments, $6 \mathrm{mM}$ of ascorbic acid, D-fructose, $\beta$-cyclodextrin, lactose and maltose were respectively used to replace glucose under identical conditions. A commercial glucose assay kit was used for the spectrophotometric determination of glucose in serum samples, and its analytical process was operated according to the manufacturer's manual (the samples of fetal bovine serum should be diluted about 20 times). All the experiments were operated in air and at room temperature without a solution purge of oxygen. Glucose solutions with concentrations of $0.51 \mathrm{mM}$ and $0.92 \mathrm{mM}$ were prepared as simulated tears of normals and diabetics, respectively.

\section{Computational modelling}

The finite difference time domain (FDTD) method was used to calculate the optical and electromagnetic properties of the $\mathrm{Ag}$ NP aggregation process. All FDTD calculations were carried out on FDTD Solutions. The input wave was defined with the totalfield scattered-field source. The observation objects were placed perpendicular to the source through the centers.

\section{Results and discussion}

Based on our proposed plasmon nanosensors combining CRAM with the LSPR effect, the polymerization of acrylic acid monomers as a means of signal amplification was utilized on the surface of the $\mathrm{Ag}$ NPs with an $\mathrm{SiO}_{2}$ coating, which is conceptually described in Fig. 1. The biocatalysis of glucose could cause the polymerization of acrylic monomers on the $\mathrm{Ag} @ \mathrm{SiO}_{2} \mathrm{NPs}$ surface, resulting in the aggregation of acrylic acid-functionalized $\mathrm{Ag} @ \mathrm{SiO}_{2}$ NPs (details in Fig. S1†). The LSPR peak position of Ag NPs varies with the change in distance between Ag NPs, which could be used as an output signal, due to the high sensitivity to the dielectric constant of the surrounding environment caused by the plasma effect (details in Fig. S1 $\dagger$ ). Owing to the chemical instability and tendency towards aggregation of Ag NPs, it is necessary to utilize a desirable surface chemistry modification to prevent the aggregation of the Ag NPs while facilitating further functionalization. The well-established surface chemistry has indicated that various surface functional group (e.g. $-\mathrm{OH},-\mathrm{NH}_{2}$, or $-\mathrm{COOH}$ ) could be covalently introduced onto the silica surface using readily available coupling agents. ${ }^{32,33}$ Therefore, a silica shell would be firstly incorporated as a spacer layer to cover the

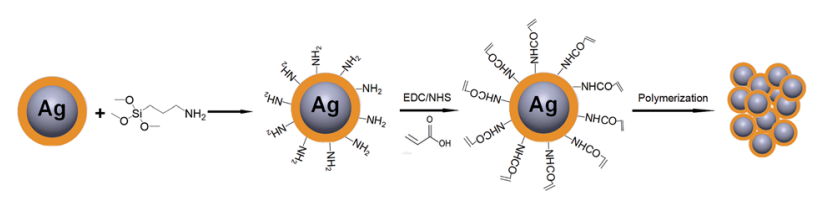

Fig. 1 Conceptual depiction of detection using polymerization-based signal amplification.
Ag core. Afterwards, the acrylic acid monomers were introduced onto the silica surface. The glucose sensing was conducted in the presence of $\mathrm{FeCl}_{2}$ salt and GOx. The glucose detection mechanism of the proposed sensor was based on free-radical polymerization by biocatalytic initiation, which is explained in the following reaction steps (eqn (1) and (2)):

$$
\begin{gathered}
\text { GOx }+ \text { glucose gluconic acid }+\mathrm{H}_{2} \mathrm{O}_{2} \\
\mathrm{Fe}^{\mathrm{II}}+\mathrm{H}_{2} \mathrm{O}_{2} \mathrm{Fe}^{\mathrm{III}}+\cdot \mathrm{OH}+\mathrm{HO}^{-}
\end{gathered}
$$

The glucose was catalyzed by GOx to produce $\mathrm{H}_{2} \mathrm{O}_{2}$ (eqn (1)), which was subsequently reduced by $\mathrm{Fe}^{\mathrm{II}}$ ions to yield $\cdot \mathrm{OH}$ free radicals (eqn (2)). The $\cdot \mathrm{OH}$ radicals initiated the polymerization of acrylic acid monomers on the silica surface and changed the LSPR resonance wavelength recorded by the UV-VIS-NIR spectrophotometer. The technique of electron spin resonance (ESR) was used to confirm the existence of $\cdot \mathrm{OH}$ after the addition of $\mathrm{Fe}^{\mathrm{II}}$ in the glucose detection. It was obviously observed that the $\mathrm{DMPO} / \cdot \mathrm{OH}$ signal intensity of the ESR spectra in the presence of $\mathrm{Fe}^{\mathrm{II}}$ was higher than that without $\mathrm{Fe}^{\mathrm{II}}$ or with acrylic acid-functionalized $\mathrm{Ag} @ \mathrm{SiO}_{2} \mathrm{NPs}$, which exhibit an intensity ratio of $1: 2: 2: 1$ from Fig. S2 (ESI $\dagger$ ). The results showed that $\cdot \mathrm{OH}$ actually existed in the system of glucose detection, which could induce the aggregation of the acrylic acid-functionalized Ag@SiO $\mathrm{NPs}_{2}$

In general, the polymerization led to the coupling of $\mathrm{Ag}$ NPs. The thinner the silica shell was, the more sensitive the plasmonic nanosensor would be. However, if the silica shell was too thin, the spacer could not efficiently protect the Ag NPs from aggregation when $\mathrm{Fe}^{\mathrm{II}}$ ions were added..$^{34}$ The thickness of the silica shell could be readily adjusted to optimize the sensitivity of the plasmonic nanosensor and the optimal thickness of the silica shell is about $10 \mathrm{~nm}$. Herein, the thickness of the silica shell as a spacer layer should be adjusted to make the plasmonic nanosensor more sensitive when the distance between the Ag NPs changed. For a typical silica coating, a freshly prepared citrate-stabilized Ag colloid was safely transferred into

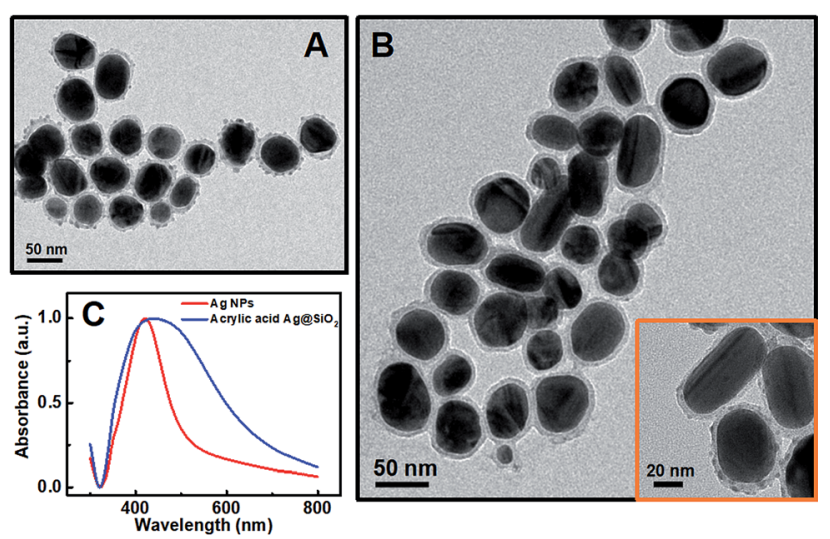

Fig. 2 (A) TEM image of $\mathrm{AgCSiO}_{2} \mathrm{NPs}$ with a shell thickness of $\mathrm{ca}$. $10 \mathrm{~nm}$. (B) Low- and high-magnification (insert) TEM images of acrylic acid-functionalized $\mathrm{Ag} \mathrm{aSiO}_{2}$ NPs with a shell thickness of ca. $10 \mathrm{~nm}$. (C) UV-visible spectra of Ag NPs (red) and acrylic acid-functionalized $\mathrm{AgCSiO}_{2} \mathrm{NPs}$ (blue). 
ethanol followed by intermittent addition of TEOS solution. The TEM images (Fig. 2A) show that the obtained Ag@SiO $\mathrm{NPs}_{2}$ had a silica coating layer of $c a .10 \mathrm{~nm}$ on the surface of the Ag NPs when $4 \mathrm{~mL}$ of $10 \mathrm{mM}$ TEOS ethanol solution was used for synthesis. A relatively rough silica surface was observed, which was attributed to the short particle radius $\left(R^{-1}\right)$, while the rough surface of the Ag@SiO $\mathrm{SPs}_{2}$ would not affect our experimental result. ${ }^{35}$ Subsequently, in order to retain the thickness of the silica coating layer, each particle was coated only with the desirable APTMS layer used in the functionalization step, assuming one APTMS molecule to occupy $0.4 \mathrm{~nm}^{2}$ on the nanoparticle surface. ${ }^{36}$ The ethanol solution of APTMS $(1 \mathrm{~mL}$, $10 \mathrm{mM}$ in ethanol) was added to $50 \mathrm{~mL}$ of $\mathrm{Ag} @ \mathrm{SiO}_{2}$ solution with vigorous stirring. Before being grafted onto the $\mathrm{Ag@SiO}$ NPs, the acrylic acid should be activated by EDC/NHS and incubated for at least $2 \mathrm{~h}$ for efficient activation. The surfacegrafted amine groups of the silica shell reacted with the activated carboxyl groups of acrylic acid to form an ester group, eventually yielding acrylic acid-functionalized $\mathrm{Ag} @ \mathrm{SiO}_{2} \mathrm{NPs}$. As shown in Fig. 2B, there was no significant change in the thickness of the spacer layer for acrylic acid-functionalized $\mathrm{Ag} @ \mathrm{SiO}_{2}$ compared with the $\mathrm{Ag} @ \mathrm{SiO}_{2} \mathrm{NPs}$ (Fig. 2A). An obvious red-shift of $c a .14 \mathrm{~nm}$ and a broadened half-peak width in the plasmon resonance band of acrylic acid-functionalized $\mathrm{Ag} @ \mathrm{SiO}_{2}$ NPs could be observed in the UV-visible spectra (Fig. 1C) as compared to those of Ag NPs (ca. $50 \mathrm{~nm}$ in diameter, see Fig. S3 in ESI $\dagger$ ), due to the increased refractive index of the surrounding medium from the Ag NPs. ${ }^{25}$

Since there is no absorption for other species (GO, GOx, $\mathrm{Fe}^{\mathrm{II}}$ ions) in our sensor system in the wavelength range of 300$800 \mathrm{~nm}$, the variations in the plasmon resonance of acrylic acid-

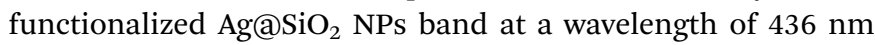
were selected as the sensor response signal (Fig. S4, see ESI $\dagger$ ). For this proposed sensing system, the variation in plasma resonance should be caused by glucose alone; therefore, reference tests without glucose were carried out under identical conditions to confirm our supposition. Within the observation time (10 $\mathrm{min})$, no spectral variation was found, which indicated that the variation in the spectrum was not affected by different concentrations of $\mathrm{FeCl}_{2}$ and was actually caused by the glucoseinduced polymerization (see Fig. S5 in ESI $\dagger$ ).

The reaction initiated by redox was influenced by the components of the glucose, $\mathrm{FeCl}_{2}$ salt and monomer concentration in this reaction system. In particular, the $\mathrm{Fe}^{\mathrm{III}}$ ions would compete with the acrylic monomer for $\cdot \mathrm{OH}$ radicals in the case of too high a concentration of $\mathrm{FeCl}_{2}$ salt in the reaction system, leading to a decrease in the polymerization efficiency. However, a low concentration of $\mathrm{FeCl}_{2}$ salt would result in too slow polymerization, which could pose difficulty in detecting the variation in the spectrum over a suitable time range. Aiming to find an optimal concentration of $\mathrm{FeCl}_{2}$ and an appropriate observation time, we varied the dosage of $\mathrm{FeCl}_{2}$ from 2 to $20 \mu \mathrm{L}$ into the $6 \mathrm{mM}$ glucose solution to perform a series of measurements. Subsequently, the results of the UV-vis spectra (see Fig. S6 in ESI $\dagger$ ) have shown that the absorption intensity at $436 \mathrm{~nm}$ showed a rapid decay with an increase in the dosage of $\mathrm{FeCl}_{2}$ (from 4 to $10 \mu \mathrm{L}$ ). It is well known that the rate of redox initiating reaction would be faster with an increase of the dosage of $\mathrm{FeCl}_{2}$, leading to acceleration of the polymerization, while the polymerization would induce the aggregation of Ag NPs and thereby cause a decrease in the plasmon resonance intensity. But, the absorption from adding $2 \mu \mathrm{L}$ of $\mathrm{FeCl}_{2}$ showed no significant change within the testing time of $40 \mathrm{~min}$. This is because too small an amount of initiator $\left(\mathrm{FeCl}_{2}\right)$ causes a very slow polymerization process (see Fig. S6A in ESI $\dagger$ ). On the other hand, the spectral change of each glucose concentration (see Fig. S6F in ESI $\dagger$ ) would be insignificant after adding too much $\mathrm{FeCl}_{2}(20 \mu \mathrm{L})$, which was ascribed to a too fast polymerization reaction that led to little difference in degree of polymerization in each sample. Finally, considering that it was a typical time range for glucose blood tests, we have selected $3 \mathrm{~min}$ as the reaction time. Meanwhile, an appropriate dosage of $10 \mu \mathrm{L} \mathrm{FeCl}_{2}$ was chosen for the test.

Our proposed mechanism of glucose detection by acrylic acidfunctionalized $\mathrm{Ag} @ \mathrm{SiO}_{2}$ NPs was that the polymerization would lead to the coupling of Ag NPs, and consequently decrease the intensity of dipolar resonance. Hence, the sensor response as a function of glucose concentration around the human physiological range in blood concentration (the normal value in humans is $6.0 \mathrm{~mm}$ ) was plotted in Fig. 3, which clearly shows a linear dependence of absorbance band maximum versus glucose concentration with a coefficient of 0.995 (Fig. 3B). The photographs of the color variation with different glucose concentrations in the plasmonic sensing devices are shown in Fig. S7. $\dagger$ Additionally, as shown in Fig. 3C, the TEM image of $\mathrm{Ag} @ \mathrm{SiO}_{2} \mathrm{NPs}_{\mathrm{f}}$ after polymerization could further confirm our mechanism that the polymerization actually caused the aggregation of Ag NPs, resulting in a reduction in the intensity of dipolar resonance.

According to a previous report, along with the aggregation of Ag NPs, the dipolar resonance from the Ag NPs at a wavelength of $420 \mathrm{~nm}$ would generally decrease in intensity and undergo a slight red shift. In addition, a new multipolar resonance would initially appear at a longer wavelength due to coupling between the Ag NPs. ${ }^{37}$ However, only the decrease in the dipolar resonance intensity could be observed without any multipolar resonance at a longer wavelength. Such a phenomenon could be attributed to
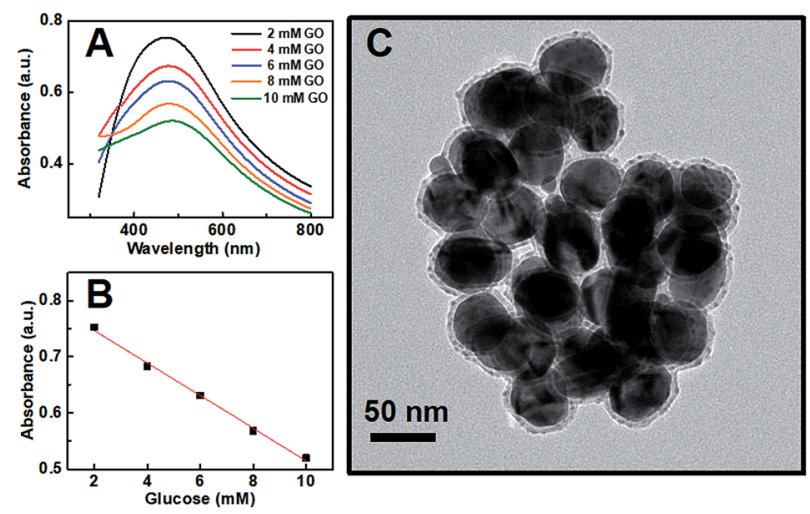

Fig. 3 (A) Representative LSPR spectra obtained from the plasmonic sensing device with different glucose concentrations: 2 (black), 4 (red), 6 (blue), 8 (orange), $10 \mathrm{mM}$ (green). (B) Absorbance band maximum versus glucose concentration. (C) The TEM image of $\mathrm{AgCSiO}_{2} \mathrm{NPs}$ after polymerization. 
two reasons as follows: first, the Ag NPs were coated with a silica shell as an outside spacer that protected the Ag NPs from severe aggregation. Second, the reaction time $(3 \mathrm{~min})$ of the glucose detection might be too short to generate the new resonance, which could not reach the degree of aggregation.

Moreover, FDTD simulation was implemented to further confirm our experimental results. In order to simplify the calculation system, the FDTD simulation calculated the plasmon resonance peak of ten $\mathrm{Ag}$ NPs with various degrees of aggregation. With the degree of aggregation increasing, the clusters in the system would gradually decrease. Simultaneously, the $\mathrm{N}$ would be set to characterize the degree of aggregation, which could be defined as the ratio of the total amount of Ag NPs to the number of clusters $\left(N=n_{\mathrm{Ag} \text { NPs }} /\right.$ $n_{\text {clusters }}$ ). According to Fig. $4 \mathrm{~A}-\mathrm{E}$, the values of $\mathrm{N}$ were enhanced from 1 to 1.67 with an increase in the degree of aggregation in addition, Fig. $4 \mathrm{~F}$ presents the simulation results for the intensity of the plasmon resonance peak. Clearly, the intensity of the extinction gradually decreased with an increase in the degree of aggregation, in line with our aforementioned experiment.

In a practical application, other species in the analytical sample should not disturb the signal, which is a typical concern for the operation of biosensors. Therefore, a series of reference experiments using other common interfering species, such as ascorbic acid, D-fructose, $\beta$-cyclodextrin, lactose and maltose instead of glucose, have been conducted under identical conditions. And there were no signals detected (see Fig. S8 in ESI $\dagger$ ), showing high selectivity toward glucose detection. Additionally, another concern for practical application is repeatability. Therefore, 10 sensors were prepared in the same way to detect a glucose solution with a concentration of $6 \mathrm{mM}$ (see
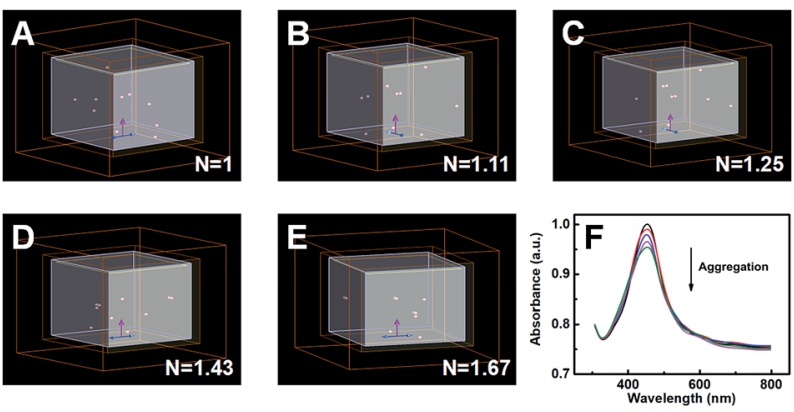

Fig. 4 (A) $-(E)$ FDTD model of different degrees of aggregation. $N$ is defined as the particle count divided by the cluster count. The particle count is set to 10 . With an increase in the degree of aggregation, the cluster count decreases from 10 to 6 . (F) The intensity of the absorption of different degrees of aggregation.

Table 1 Determination of blood glucose in fetal bovine serum samples

\begin{tabular}{lll}
\hline Samples & $\begin{array}{l}\text { Plasmonic } \\
\text { biosensor }\end{array}$ & $\begin{array}{l}\text { Commercial glucose } \\
\text { assay kit }\end{array}$ \\
\hline 1. & $6.0 \pm 0.2 \mathrm{mM}$ & $6.0 \pm 0.1 \mathrm{mM}$ \\
2. & $6.0 \pm 0.2 \mathrm{mM}$ & $6.0 \pm 0.1 \mathrm{mM}$ \\
3. & $6.1 \pm 0.3 \mathrm{mM}$ & $5.9 \pm 0.2 \mathrm{mM}$
\end{tabular}

Table 1), which showed that the sensors had good repeatability with a standard deviation of $2.5 \%$.

To further verify the practicability of the plasmonic nanosensor, it was used for the detection of the glucose concentration in fetal bovine serum and compared with a commercial glucose assay kit (see Fig. S9 in ESI $\dagger$ ). According to Table 1, the performance of our plasmonic nanosensor could be comparable to the commercial glucose assay kit, indicating that the proposed nanosensor is suitable for practical applications. In addition, solutions of simulated tears (ca. $0.92 \mathrm{mM}$ for diabetics, ca. $0.51 \mathrm{mM}$ for normals $)^{38}$ were prepared to further investigate the practicability of the plasmonic nanosensor (Table S1 and Fig. S10†). In addition, the LOD of our plasmonic nanosensor could reach $2.06 \times 10^{-5} \mathrm{M}, 10$ times lower than the limit of the commercial glucose assay kit of $1.1 \times 10^{-4} \mathrm{M}$. The developed approach can not only be used in a blood glucose monitor, but can also be utilized as a convenient tool to detect very low concentrations of glucose in solution.

\section{Conclusions}

In summary, acrylic acid-functionalized $\mathrm{Ag} @ \mathrm{SiO}_{2}$ NPs were rationally designed as a plasmonic nanosensor to obtain highly sensitive and fast colorimetric glucose detection with less monomer consumption, which effectively integrated CRAM with the LSPR effect, developing CRAM at the molecular scale. As a result, the limit of detection of our plasmonic nanosensor could reach $2.06 \times 10^{-5} \mathrm{M}, 10$ times lower than a commercial glucose assay kit with a limit of $1.1 \times 10^{-4} \mathrm{M}$. Meanwhile, FDTD simulation has been used to confirm our purposed mechanism of detection, which was that the intensity of the LSPR peak gradually decreases with an increase in the degree of aggregation of Ag NPs. Therefore, the study could potentially be used in a blood glucose monitor and would be suitable for other practical applications for the detection of low concentrations of glucose.

\section{Conflicts of interest}

There are no conflicts to declare.

\section{Acknowledgements}

This work was supported by the National Natural Science Foundation of China (Grant No. 51833011) and Guangdong YangFan Innovative \& Entepreneurial Research Team Program (Project No. 201633002).

\section{Notes and references}

1 O. J. Mace, J. Affleck, N. Patel and G. L. Kellet, J. Physiol., 2007, 582, 379-392.

2 U. Schlemmer, W. Frølich, R. M. Prieto and F. Grases, Mol. Nutr. Food Res., 2009, 53, S330-S375.

3 Z. C. Chen and C. J. Zhong, Prog. Neurobiol., 2013, 108, 21-43.

4 Y. Xiao, F. Patolsky, E. Katz, J. F. Hainfeld and I. Willner, Science, 2003, 299, 1877-1881. 
5 L. Bahshi, R. Freeman, R. Gill and I. Willner, Small, 2009, 5, 676-680.

6 Q. Liu, Y. Yang, H. Li, R. Zhu, Q. Shao, S. Yang and J. Xu, Biosens. Bioelectron., 2015, 64, 147-153.

7 Q. Liu, Y. Yang, X. Lv, Y. Ding, Y. Zhang, J. Jing and C. Xu, Sens. Actuators, B, 2017, 240, 726-734.

8 M. Chen, B. Yang, J. Zhu, H. Liu, X. Zhang, X. Zheng and Q. Liu, Mater. Sci. Eng., C, 2018, 90, 610-620.

9 K. Wu, X. Zhao, M. Chen, H. Zhang, Z. Liu, X. Zhang, X. Zhu and Q. Liu, New J. Chem., 2018, 42, 9578-9587.

10 H. Liu, Y. Ding, B. Yang, Z. Liu, Q. Liu and X. Zhang, Sens. Actuators, B, 2018, 271, 336-345.

11 Y. Ding, B. Yang, H. Liu, Z. Liu, X. Zhang, X. Zheng and Q. Liu, Sens. Actuators, B, 2018, 259, 775-783.

12 O. Lyandres, N. C. Shah, C. R. Yonzon, J. T. Walsh, M. R. Glucksberg and R. P. Van Duyne, Anal. Chem., 2005, 77, 6134-6139.

13 L. J. Xu, Z. C. Lei, J. Li, C. Zong, C. J. Yang and B. Ren, J. Am. Chem. Soc., 2015, 137, 5149-5154.

14 L. J. Xu, C. Zong, X. S. Zheng, P. Hu, J. M. Feng and B. Ren, Anal. Chem., 2014, 86, 2238-2245.

15 J. R. Adleman, D. A. Boyd, D. G. Goodwin and D. Psaltis, Nano Lett., 2009, 9, 4417-4423.

16 W. E. I. Sha, H. L. Zhu, L. Z. Chen, W. C. Chew and W. C. H. Choy, Sci. Rep., 2015, 5, 8525-8533.

17 L. Ma, S. Liang, X. L. Liu, D. J. Yang, L. Zhou and Q. Q. Wang, Adv. Funct. Mater., 2015, 25, 898-904.

18 J. Homola, S. S. Yee and G. Gauglitz, Sens. Actuators, B, 1999, 54, 3-15.

19 Z. Li, X. Miao, Z. Cheng and P. Wang, Sens. Actuators, B, 2017, 243, 731-737.

20 X. Miao, Z. Cheng, H. Ma, Z. Li, N. Xue and P. Wang, Anal. Chem., 2018, 90, 1098-1103.

21 P. Alivisatos, Nat. Biotechnol., 2004, 22, 47-52.
22 B. Nikoobakht and M. A. El-Sayed, Chem. Mater., 2003, 15, 1957-1962.

23 W. Hong, Y. Zhang, L. Gan, X. Chen and M. Zhang, J. Mater. Chem. C, 2015, 3, 6185-6191.

24 G. Chen, D. Wang, W. Hong, L. Sun, Y. Zhu and X. Chen, Small, 2017, 1602612.

25 P. Mulvaney, Langmuir, 1996, 12, 788-800.

26 H. D. Sikes, R. R. Hansen, L. M. Johnson, R. Jenison, J. W. Birks, K. L. Rowlen and C. N. Bowman, Nat. Mater., 2008, 7, 52-56.

27 B. J. Berron, L. M. Johnson, X. Ba, J. D. McCall, N. J. Alvey, K. S. Anseth and C. N. Bowman, Biotechnol. Bioeng., 2011, 108, 1521-1528.

28 C. Li, H. Wang, J. Shen and B. Tang, Anal. Chem., 2015, 87, 4283-4291.

29 B. J. Berron, A. M. May, Z. Zheng, V. Balasubramaniamn and C. N. Bowman, Lab Chip, 2012, 12, 708-710.

30 S. Liu, Z. Zhang and M. Han, Anal. Chem., 2005, 77, 25952600.

31 P. C. Lee and D. Meisel, J. Phys. Chem., 1982, 86, 3391-3395.

32 R. K. Iler, The chemistry of silica, Wiley-Interscience, New York, 1979.

33 R. D. Badley, Langmuir, 1990, 6, 792-801.

34 H. G. Han, J. Goeb, Z. D. Lu and Y. D. Yin, Langmuir, 2011, 27, 5282-5289.

35 C. Graf, D. L. J. Vossen, A. Imhof and A. V. Blaaderen, Langmuir, 2003, 19, 6693-6700.

36 F. J. Boerio, L. Armogan and S. Y. Cheng, J. Colloid Interface Sci., 1980, 73, 416-424.

37 I. Blakey, Z. Merican and K. J. Thurecht, Langmuir, 2013, 29, 8266-8274.

38 D. K. Sen and G. S. Sarin, Br. J. Ophthalmol., 1980, 64, 693695. 